This item was submitted to Loughborough's Research Repository by the author.

Items in Figshare are protected by copyright, with all rights reserved, unless otherwise indicated.

\title{
Advances in disturbance/uncertainty estimation and attenuation [Guest editors' introduction]
}

PLEASE CITE THE PUBLISHED VERSION

http://dx.doi.org/10.1109/TIE.2015.2453347

PUBLISHER

(C) IEEE

VERSION

AM (Accepted Manuscript)

\section{PUBLISHER STATEMENT}

This work is made available according to the conditions of the Creative Commons Attribution-NonCommercialNoDerivatives 4.0 International (CC BY-NC-ND 4.0) licence. Full details of this licence are available at: https://creativecommons.org/licenses/by-nc-nd/4.0/

\section{LICENCE}

CC BY-NC-ND 4.0

\section{REPOSITORY RECORD}

Chen, Wen-Hua, Kouhei Ohnishi, and Lei Guo. 2016. "Advances in Disturbance/uncertainty Estimation and Attenuation [guest Editors' Introduction]”. figshare. https://hdl.handle.net/2134/20483. 


\section{Advances in Disturbance/Uncertainty Estimation and Attenuation}

T HERE is even increasing desire on achieving high precision control for new generations of mechatronics and robotics, electrical machines and drives, power electronics, energy conversion, aerospace and automotive systems. However disturbances and uncertainties caused by the changes of physical systems of concern or their operational environment are widely present. As is well known, there are a number of conflicts/constraints in control system design (e.g. tracking vs disturbance rejection, and the nominal performance vs. robustness). Disturbance observer and related techniques provide a flexible framework in dealing with these challenges since 1983. In this group of techniques, an observer or alike is designed to estimate unknown external disturbance and (or) the influence of unmodelled dynamics/uncertainties. By the appropriate use of the outcome of the observer, disturbance rejection and robustness of an existing control system could be significantly improved. As such, disturbance and uncertainty estimation techniques play the core role in this kind of control algorithms. These control schemes are able to not only explicitly exploit the modelling information in control system design as many other control design methods, but also cope with unknown disturbances, unmodelled dynamics and various variations/uncertainties without scarifying the nominal performance.

The main objective of this Special Section on "Advances in Disturbance/Uncertainty Estimation and Attenuation " is to bring the ideas of the worldwide research community working on various disturbance/uncertainty estimation and rejection methods in different engineering sectors into common platform, and to present the latest advances and developments in design methods for disturbance/uncertainty estimation, design methods for disturbance/uncertainty attenuation, stability and performance analysis, practical implementation, and various case studies of the applications of these techniques.

Twenty one papers were eventually selected from a large amount of submissions through a strict peer-reviewed procedure, which can represent the most recent progress in disturbance/uncertainty estimation and attenuation, and their applications. Despite of different focuses, almost all papers cover both theoretic developments and applications. Actually most of the papers verify the design and analysis through experimental tests. All the main design methods in the disturbance/uncertainty estimation and attenuation have been covered by the selected papers. From the application point of view, the selected contributions cover power electronics (e.g. DCDC converts), motor and drive systems (e.g. linear reluctance motors, rotary actuators), magnetic levitation systems, photovoltaic generators, haptics for medical training or surgery, robotic exoskeleton/manipulators, chemical rectors, various aerospace vehicles, and gasoline engines. These applications spread in a quite broad range of industrial sectors including electrical, mechanical, automotive and aerospace engineering, energy, transport and health care.

Due to the diversity of applications and methodologies used in the selected papers, it is not easy to group them. To make them easy for readers' reference, the selected contributions are grouped in terms of methodologies used in the development and (or) applications. Brief summaries of the selected papers are give as follows.

\section{A. Disturbance observer based technique}

Not surprisingly, as disturbance observer based control is the widely used method, this is the largest group of the selected contributions. There are two subgroups in this category: classic frequency domain design methods and time domain (state space) design methods.

There are four papers devoted to classic frequency domain disturbance observer design. In reference [1], authored by $\mathrm{Li}$ et al., the problem of robotic exoskeleton control is considered based on disturbance estimation technique with the aim to removing built-in torque sensing units. To this end, fuzzy approximation and disturbance observers are utilized to estimate and compensate the disturbance torques caused by viscous friction, gravity, payloads and unknown input saturation. Then extensive experiments are conducted on the upper limb exoskeleton to illustrate the performance.

The paper [2], authored by Sitbon et al., applies the frequency-domain disturbance observer technique to the regulation problem of terminal voltage of photovoltaic generators. Experimental results show that incorporating a disturbance observer into the nominal controller can achieve satisfactory control performance for the whole operation range, even though the nominal controller is only designed and valid for a single nominal operating point. While, reference [3], authored by Nagatsu and Katsura, applies the disturbance observer technique to motion reproduction, where environmental variances between the motion saving and reproduction phases are treated as disturbances. Experiment results show that the resulted two degree of freedom control system can achieve higher control precision in comparison with traditional one degree of freedom control approach.

The work [4], authored by Onoyama and Katsura, shows a mixed reproduction of physical human operation and databased motions, where two indices for human interaction under automatic reproduction of the saved motion were introduced including human operationality and data-based motion reproducibility. These two indices are stabilized based on the frequency filtering developed in the paper, where the effectiveness is confirmed by experimental results in comparison with the conventional methods. 
Three selected papers are devoted to disturbance observer based control using state space approaches. The work [5], authored by Yang, et al., addresses the predication accuracy enhancement problem using continuous-time model predictive control and a disturbance observer. The disturbance estimate is introduced in the output prediction, which leads to the desired offset-free tracking performance. Compared with the existing approaches, the proposed approach requires smaller computational burden and results in nominal performance recovery property. Experimental results of a DC-DC buck converter system validate the effectiveness of the proposed algorithm.

The paper [6], authored by Ginoya et al., proposes a delta operator based discrete-time disturbance observer for a class of uncertain systems, which can not only estimate the disturbances but also their derivatives. Own to the use of the delta operator, the proposed design technique unifies the design of continuous-time and discrete-time time disturbance observers. The proposed disturbance observer design technique is applied to deal with mismatched disturbance rejection in conjunction with a discrete-time sliding mode controller with the experimental validation on a 2-DOF serial flexible joint manipulator.

The work [7], authored by Ding, deal with consensus disturbance rejection of network-connected dynamic systems using disturbance observers with the application in swarm of unmanned aircraft systems. It extends the existing disturbance observer based control to a group of multiple dynamic systems. Different from the existing approaches, only the relative state information is used in disturbance rejection, and a fully distributed disturbance rejection control architecture is presented.

\section{B. Active Disturbance Rejection Control (ADRC)}

Reference [8], authored by Zhao et al., considers the disturbance rejection for position control of a magnetic rodless cylinder in servo systems without pressure states. Extended State Observer (ESO) is used to estimate the nonlinearity, while a tracking differentiator is used to reduce its overshoot. Experimental results validate that the active disturbance rejection control (ADRC) strategy can result in a smaller steadystate control error.

The reference [9], authored by Xue et al., proposes an adaptive ESO (AESO) where the gain of ESO can be automatically tuned such that the estimation errors of both states and "total disturbances" is further reduced. The proposed control strategy is applied to air-fuel ratio control of a gasoline engine which has significant nonlinear uncertainties due to the unknown speed change and fuel film dynamics. Experimental results demonstrate the high control precision despite the uncertainties and sensor noises.

Falling into the context of ADRC, an AESO is proposed for nonlinear system with disturbances in [10], authored by $\mathrm{Pu}$ et al. The AESO is designed as a linear time-varying form which can combine the theoretical completeness of linear ESO with practical performance of nonlinear ESO. Differential algebraic spectral theory is used to assign the time-varying PDeigenvalues of AESO error dynamics. Both stability is established and estimation error bound of the AESO is estimated in the presence of unknown disturbances. Simulation results on both numerical and practical examples are given to compare the performance of different types of ESO.

\section{Unknown Input Observers (UIO)/Uncertainty and Distur- bance Estimators (UDE)}

Motivated by the potential applications of disturbance decoupling observer design in fault diagnosis and unknown input estimation, the work [11], authored by Ichalal and Mammar, considers the design of Unknown Input Observer (UIO) for Linear Parameter Varying (LPV) systems. In comparison with the classical UIO for linear time invariant system, the contribution is twofold. First, it considers a more generic type of system (i.e., LPV) where the classic approaches may fail. Secondly, it generalizes the observer design to system with arbitrary relative degree where the classical rank conditions are no longer satisfied.

Reference [12] considers the robust control of a class of non-affine nonlinear system using Uncertainty and Disturbance Estimator (UDE), where no "inverse operator" construction is needed and consequently the control singularity problem can be avoided. In the proposed UDE based control, the assumption on the uncertainty and disturbance term is also relaxed and only its bandwidth information is required. Both simulation studies and experimental validation on a servo system with non-affine uncertainties are carried out to demonstrate its properties.

\section{Sliding mode observers and high gain observers}

The work [13], authored by Tian et al., investigates the problem of finite time tracking control of Reusable Launch Vehicle in the presence of mismatched disturbances. An adaptive multi-variable sliding mode disturbance observer is proposed to estimate the disturbances, based on which a continuous multivariate homogeneity second order sliding mode controller is designed to achieve attitude tracking in finite time. The properties of the resulted compound controller (e.g. finite time convergence, nominal performance recovery) is verified through simulation.

The work [14], authored by Ding et al., considers the second-order sliding mode control design for nonlienar systems with uncertainties bounded by positive functions. Two different second-order control algorithms are proposed including a discontinuous one and a quasi-continuous one. The discontinuous one is built based on Lyapunov method and, as a result, the global finite-time Lyapunov stability is established using the modified adding a power integrator technique. The quasi-continuous one is further developed such that the chattering phenomenon can be attenuated.

The work [15], authored by Shi et al., presents a new descriptor sliding mode observer using quantized measurements for a class of Markovian jump systems in the presence of actuator faults. A discontinuous input is synthesized to reject actuator faults by an off-line static compensation of quantization effects. The asymptotical estimation of both states and quantization errors can be achieved by the proposed 
observer. case study on an F-404 aircraft engine system is provided to show its effectiveness.

The reference [16], authored by Jiang et al., considers the decentralized fault tolerant control design for near space vehicle attitude dynamics. A multi-observer based local fault identification algorithm (including fault detection observer, fault parameters identification observers, and associated decision making mechanism) is proposed to identify actuator faults. A global adaptive sliding mode observer technique is used to design the command filter backstepping fault tolerant controller. Case study on a near space vehicle shows that the proposed fault tolerant control scheme can accommodate actuator faults, control surface damages, uncertainties and disturbances simultaneously.

Motivated by sensorless control in automotive applications, the paper [17] proposes a two-stage observer structure to cope with uncertainties and external disturbance. It combines a high gain observer with a sliding mode observer by exploiting the strengths of each of them. It has shown that the high gain observer can effectively estimate and compensate the influence of the uncertainties in the electromagnetic valve actuators and unmeasurable disturbances from burning phase of the engine and external torque while the sliding mode observer improves the estimation by achieving zero estimation error in a finite time. Laboratory experimental tests demonstrate the proposal scheme performs favorably over the existing Kalman filtering based method.

\section{E. Practical/emerging estimation techniques}

For a specific class of systems or for a specific application, a tailored design method for disturbance and uncertainties estimation/attenuation could be developed. In real applications, a single friction model is usually insufficient to describe friction behaviour due to large uncertainties, the work [18], authored by Ruderman and Iwasaki, proposes a new nonlinear friction observer to achieve an accurate friction estimation without the use of a specific dynamic friction model. The proposed friction observer is then applied to a rotary actuator system, and experimental results show that precise positioning can be achieved by incorporating the friction observer.

Considering the fact that unknown measurement delays usually degrade system performance under output feedback control, the work [19], authored by Z. Gao, develops an approach to attenuate their adverse effect for discrete-time Lipschitz nonlinear systems by estimating and compensating them through an augmented observer. The constant piecewise disturbance decoupling problem is also considered, which can also reduce the adverse effect of disturbance in conjunction with measurement delays.

In reference [20], authored by Yu et al., adaptive fuzzy output feedback control is considered for a class of strict-feedback systems with parametric uncertainties and unmeasured states using the observer and command filter. Fuzzy logic systems are used to approximate the unknown and nonlinear functions and fuzzy state observer is to estimate the unmeasurable states. On this basis, command filtered backstepping control is designed to avoid the explosion of complexity in the backstepping de- sign, and then compensating signals are introduced to remove the effect of the errors caused by command filters.

The work [21], authored by Zhang et al., considers an exponentially tracking control problem for a magnetic leviation system with unidirectional input constraint in the presence of parameter uncertainties and external disturbances. Input transformation and augmented dynamics are adopted to generate a virtual control input so as to remove the unidirectional constraint. Auxiliary filters are developed to estimate disturbances. Both numerical simulation and experimental tests on a magnetic leviation system are carried out to validate the effectiveness of the proposed algorithm.

Our overview of the selected twenty one papers in Special Section on "Advances in Disturbance/Uncertainty Estimation and Attenuation" has been completed. The Guest Editors expect that this Special Section will improve the awareness of the research and industrial community about this group of methods, and promote the further research and applications in this area, particularly cross fertilisation of different strategies in this area and with other related well established methods. We believe that with all the combined effort, significant advances will be made in both theoretic developments and applications, and disturbance/undertabty estimation and attenuation technique will become one of powerful tools available for engineers in dealing with disturbance and uncertainties to fulfil increased performance requirements in motion control and other areas.

\section{GUEST EDITORS}

Wen-Hua Chen, Department of Aeronautical and Automotive Engineering, Loughborough University, Loughborough, the United Kingdom

Kouhei Ohnishi,Department of System Design Engineering, Keio University, Yokohama, Japan

Lei Guo, School of Automation and Electronic Engineering, Beihang University, Beijing, China

\section{ACKNOWLEDGMENT}

The Guest Editors would like to express their deep gratitude to all the authors that have submitted their valuable contributions and to the numerous and highly qualified anonymous reviewers. We think that the selected contributions, which represent the current state of the art in the field, will be of great interest to the industrial electronics community. They would like to thank Prof. Carlo Cecati, Editor-in-Chief of the IEEE TRANSACTIONS IN INDUSTRIAL ELECTRONICS, for giving us the opportunity to organize this Special Section and for all the encouragement, help and support given throughout the process, and Ms. Sandra McLain for her professional support and assistance during the whole preparation of this Special Section. 
This is the author's version of an article that has been published in this journal. Changes were made to this version by the publisher prior to publication.

The final version of record is available at http://dx.doi.org/10.1109/TIE.2015.2453347

\section{REFERENCES}

[1] Z. Li, C. Su, L. Wang, Z. Chen and T. Chai, "Nonlinear Disturbance Observer Based Control Design for a Robotic Exoskeleton Incorporating Fuzzy Approximation," IEEE Trans. Ind. Electron., vol. xx, no. yyyy, pp. Xxxx-Xxxx, Month. ZZZZ.

[2] M. Sitbon, S. Schacham and A. Kurperman, "Disturbance Observer Based Voltage Regulation of Current-Mode-Boost-Converter-Interfaced Photovoltaic Generator," IEEE Trans. Ind. Electron., vol. xx, no. yyyy, pp. $\mathrm{xxxx}-\mathrm{xxxx}$, Month. ZZZZ.

[3] Y. Nagatsu and S. Katsura, "Design Strategies for Motion Reproduction Based on Environmental Disturbance Compensation, "IEEE Trans. Ind. Electron., vol. xx, no. yyyy, pp. xxxx-xxxx, Month. ZZZZ.

[4] H. Onoyama and S. Katsura, "Mixed Reproduction of Physical Human Operation and Data-based Motions by Frequency Filtering, "IEEE Trans. Ind. Electron., vol. xx, no. yyyy, pp. xxxx-xxxx, Month. ZZZZ.

[5] J. Yang, W.X. Zheng, S.H. Li, B. Wu and M. Cheng "Design of A Prediction Accuracy Enhanced Continuous-Time MPC for Disturbed Systems Via A Disturbance Observer," IEEE Trans. Ind. Electron., vol. xx, no. yyyy, pp. xxxx-xxxx, Month. ZZZZ.

[6] D. Ginoya, P.D. Shendge and S.B. Phadke "Delta operator based extended disturbance observer and its applications, "IEEE Trans. Ind. Electron., vol. xx, no. yyyy, pp. xxxx-xxxx, Month. ZZZZ.

[7] Z. Ding "Consensus Disturbance Rejection with Disturbance Observers, " IEEE Trans. Ind. Electron., vol. xx, no. yyyy, pp. xxxx-xxxx, Month. ZZZZ.

[8] L. Zhao, Y. Yang, Y. Xia and Z. Liu, "Active Disturbance Rejection Position Control For a Magnetic Rodless Pneumatic Cylinder, "IEEE Trans. Ind. Electron., vol. xx, no. yyyy, pp. xxxx-xxxx, Month. ZZZZ.

[9] W. Xue, W. Bai, S. Yang, K. Song, Y. Huang and H. Xie, "ADRC with Adaptive Extended State Observer and Its Application to Air-fuel Ratio Control in Gasoline Engines, "IEEE Trans. Ind. Electron., vol. xx, no. yyyy, pp. xxxx-xxxx, Month. ZZZZ.

[10] Z. Pu, R. Yuan, J. Yi and X. Tan, "A Class of Adaptive Extended State Observers for Nonlinear Disturbed Systems, "IEEE Trans. Ind. Electron., vol. xx, no. yyyy, pp. xxxx-xxxx, Month. ZZZZ.

[11] D. Ichalal and S. Mammar, "On Unknown Input Observers For LPV Systems, "IEEE Trans. Ind. Electron., vol. xx, no. yyyy, pp. xxxx-xxxx, Month. ZZZZ.

[12] B. Ren, Q. C. Zhong and J. Chen, "Robust Control for a Class of Nonaffine Nonlinear Systems Based on the Uncertainty and Disturbance," IEEE Trans. Ind. Electron., vol. xx, no. yyyy, pp. $\mathrm{xxxx}-\mathrm{xxxx}$, Month. ZZZZ.

[13] B. Tian, L. Yin and H. Wang "Finite Time Reentry Attitude Control Based on Adaptive Multivariable Disturbance Compensation," IEEE Trans. Ind. Electron., vol. xx, no. yyyy, pp. xxxx-xxxx, Month. ZZZZ.

[14] S. Ding, J. Wang and W. Zheng "Second-order Sliding Mode Control for Nonlinear Uncertain Systems Bounded by Positive Functions, " IEEE Trans. Ind. Electron., vol. xx, no. yyyy, pp. xxxx-xxxx, Month. ZZZZ.

[15] P. Shi, M. Liu and L. Zhang "Fault-Tolerant Sliding Mode Observer Synthesis of Markovian Jump Systems Using Quantized Measurements, "IEEE Trans. Ind. Electron., vol. xx, no. yyyy, pp. xxxx-xxxx, Month. ZZZZ

[16] B. Jiang, D.Z. Xu and P. Shi "Robust NSV Fault Tolerant Control System Design Against Actuator Faults and Control Surface Damage Under Actuator Dynamics," IEEE Trans. Ind. Electron., vol. xx, no. yyyy, pp. $\mathrm{xxxx}-\mathrm{xxxx}$, Month. ZZZZ.

[17] P. Mercorelli "A Two-Stage Sliding Mode High-Gain Observer to Reduce uncertainties and Disturbances Effects for Sensorless Control in Automotive Applications, "IEEE Trans. Ind. Electron., vol. xx, no. yyyy, pp. xxxx-xxxx, Month. ZZZZ.

[18] M. Ruderman and M. Iwasaki "Observer of Nonlinear Friction Dynamics for Motion Control, "IEEE Trans. Ind. Electron., vol. xx, no. yyyy, pp. $\mathrm{xxxx}-\mathrm{xxxx}$, Month. ZZZZ.

[19] Z. Gao, "Estimation and Compensation for Lipschitz Nonlinear DiscreteTime Systems Subjected to Unknown Measurement Delays, " IEEE Trans. Ind. Electron., vol. xx, no. yyyy, pp. xxxx-xxxx, Month. ZZZZ.

[20] J. Yu, P. Shi, W. Dong and H. Yu "Observer and Command FilterBased Adaptive Fuzzy Output Feedback Control of Uncertain Nonlinear Systems, "IEEE Trans. Ind. Electron., vol. xx, no. yyyy, pp. xxxx-xxxx, Month. ZZZZ.
[21] Y. Zhang, B. Xian and S. Ma "Continuous Robust Tracking Control for Magnetic Levitation System with Unidirectional Input Constraint, " IEEE Trans. Ind. Electron., vol. xx, no. yyyy, pp. xxxx-xxxx, Month. ZZZZ.

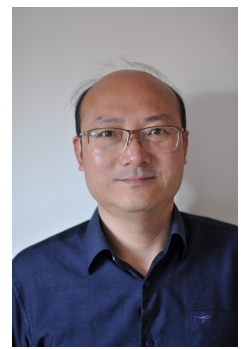

Wen-Hua Chen (M'00-SM'06) received the M.Sc. and Ph.D. degrees from Northeast University, Shenyang China, in 1989 and 1991, respectively.

From 1991 to 1996, he was a Lecturer and then Associate Professor with the Department of Automatic Control, Nanjing University of Aeronautics and Astronautics, Nanjing, China. From 1997 to 2000 , he held a research position and then a Lecturer in control engineering with the Centre for Systems and Control, University of Glasgow, Glasgow, UK. In 2000, he moved to the Department of Aeronautical and Automotive Engineering, Loughborough University, Loughborough, UK, as a Lecturer, where he was appointed as a Professor in 2012. His research interests include the development of advanced control strategies (Nonlinear Model Predictive Control, Disturbance Observer Based Control, etc.) and their applications in aerospace and automotive engineering. Currently, much of his work has also involved in the development of Unmanned Autonomous Intelligent Systems. He is a Fellow of IET.

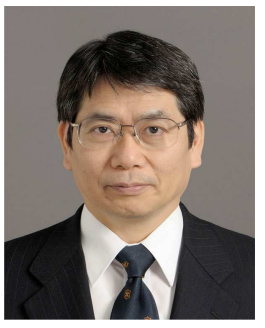

Kouhei Ohnishi (S'78-M'80-SM'00-F'01) received the B.E., M.E., and Ph.D. degrees in electrical engineering from The University of Tokyo, Tokyo, Japan, in 1975,1977 , and 1980 , respectively.

Since 1980, he has been with the Department of System Design Engineering, Keio University, Yokohama, Japan. His research interests include motion control, robotics, mechatronics, and haptics.

Dr. Ohnishi has received Best Paper Awards from the Institute of Electrical Engineers of Japan and the Japan Society for Precision Engineering, and Outstanding Paper Awards at the IEEE Industrial Electronics Society Conferences (IECON'85, IECON'92, and IECON'93). He also received the EPE-PEMC Council Award and the Dr. Ing. Eugene Mittelmann Achievement Award from the IEEE Industrial Electronics Society in 2004.

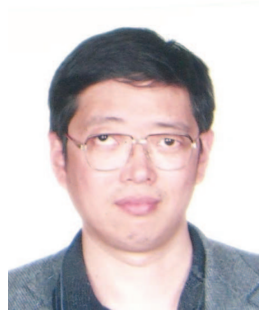

Lei Guo was born in Qufu, China, in 1966. He received his B.S. and M.S. degrees from Qufu Normal University (QFNU), China in 1988 and 1991, respectively and the Ph.D. degree in control engineering from Southeast University (SEU) in 1997.

From 1991 to 1994, he was with Qingdao University (QDU) as a lecturer. From 1997 to 1999, he was a post-doctoral fellow at Southeast University. From 1999 to 2000 , he was a research fellow in IRCCyN, Nantes, Frances. From 2000 to 2003, he was a research fellow at Glasgow University, Loughborough University and UMIST, UK. In 2004, he joined the Institute of Automation in Southeast University as a professor. In 2006 he became a professor at the School of Instrumentation and Opto-Electronics Engineering, and now at the School of Automation and Electronic Engineering, Beihang University, Beijing, China. His research interests include robust control, stochastic systems, fault detection, filter design and nonlinear control with their applications to aerospace systems.

Dr. Guo is an Awarder of the National Science Fund for Distinguished Young Scholars of China, and a Changjiang Distinguished Professor of the Ministry of Education of China. He has published more than 120 papers and one monograph and served as an Editor for 5 journals. 